\title{
Uma Plataforma Integradora da Educação no Território Inteligente
}

\author{
Douglas Rolim ${ }^{1}$, Larysse Silva ${ }^{1}$, Thais Batista ${ }^{1}$, Frederico Lopes ${ }^{2}$, \\ Everton Cavalcante ${ }^{1}$, Nélio Cacho ${ }^{1}$, Jair Leite ${ }^{1}$, Betânia Leite Ramalho ${ }^{3}$ \\ ${ }^{1}$ Departamento de Informática e Matemática Aplicada (DIMAp), \\ Universidade Federal do Rio Grande do Norte (UFRN), Natal, Brasil \\ ${ }^{2}$ Instituto Metrópole Digital (IMD), \\ Universidade Federal do Rio Grande do Norte (UFRN), Natal, Brasil \\ ${ }^{3}$ Centro de Educação (CE), \\ Universidade Federal do Rio Grande do Norte (UFRN), Natal, Brasil \\ \{douglasrolim, betania.ramalho.edu\}@gmail.com, \\ larysse.savanna@ufrn.edu.br, \\ \{thais, everton, neliocacho, jair\}@dimap.ufrn.br, fred@imd.ufrn.br
}

Resumo. Este artigo apresenta o SGeoL-Educ, uma plataforma integradora que alia georreferenciamento de dados de uma cidade a informações educacionais provenientes de diversas fontes. O SGeoL-Educ viabiliza a análise integrada, multidimensional e territorializada da educação escolar por meio de uma correlação dos dados de educação com informações do território onde as escolas estão inseridas, trazendo à tona informações que ficariam obscuras em uma visão clássica, unidimensional. A plataforma também permite visualizar informações sobre mapas, fazer consultas cruzando dados de diferentes fontes e espacializar resultados referentes a fatores de vulnerabilidades socioeducativas, notadamente aqueles mais significativos no contexto escolar sobre o território. O SGeoL-Educ foi instanciado para a cidade do Natal, Brasil, e integra informações provenientes de várias fontes: (i) dados geográficos da cidade, (ii) dados de segurança, e (iii) dados de desempenho e censo escolar fornecidos pela Secretaria de Educação do Rio Grande do Norte e pelo Instituto Nacional de Estudos e Pesquisas Educacionais (INEP).

Abstract. This paper presents SGeol-Educ, an integrating platform that combines georeferenced data of a city with educational information from several sources. SGeoL-Educ allows for an integrated, multidimensional territorialized analysis of school education through a correlation of education data with information about the territory where schools are inserted, bringing up information that would be obscure in a classic, one-dimensional view. The platform allows visualizing information on maps, making queries across data from different sources, and spatialize results regarding socio-educational vulnerability factors, especially the most significant ones in the school context over the territory. SGeoL-Educ was instantiated to the city of Natal, Brazil, and it integrates information from various sources: (i) geographical data of the city, (ii) security data, and (iii) school performance and census data provided by the Secretariat 
VII Congresso Brasileiro de Informática na Educação (CBIE 2018)

Anais do XXIX Simpósio Brasileiro de Informática na Educação (SBIE 2018)

of Education of Rio Grande do Norte and by the Brazilian National Institute for Educational Studies and Research (INEP).

\section{Introdução}

A gestão pública da educação básica (níveis infantil, fundamental e médio) requer a ampla identificação de problemas e suas causas, para que se possa intervir sobre eles com sucesso. Para alcançar esse objetivo, pode-se fazer uso de soluções que correlacionem os diversos fatores envolvidos nesse contexto, ao invés de se ter iniciativas pontuais, cada uma abordando isoladamente um determinado tipo de problema ou fator.

Os dados educacionais, que fornecem informações quantitativas referentes ao ensino e à aprendizagem, são valiosos, porém eles são insuficientes se considerados de forma isolada. Por exemplo, alguns dados que revelam limitações de aprendizagem não são usados nas atividades formativas de professores ou na melhoria dos projetos pedagógicos escolares. Para que se possa avançar na gestão da Educação, é importante envolver o máximo de atores em torno de informações que permitam análises quantitativas e qualitativas de forma ampla e contextualizada, integrando fatores não educacionais que muitas vezes interferem no processo de ensino e aprendizagem.

Este artigo apresenta uma solução dentro de um projeto mais amplo que aborda a problemática das vulnerabilidades educativas, sociais, econômicas e demográficas, dentre outras, que podem influenciar no impacto da educação escolar na formação discente nos ambientes escolares públicos brasileiros. A principal premissa desse projeto é que tais vulnerabilidades não devem ser analisadas de forma unidimensional, que corresponde à visão clássica dos gestores em que se analisa a qualidade do ensino observando tão somente os resultados das avaliações realizadas pelo Ministério da Educação (MEC). Em vez de fazer uso dessa visão unidimensional, que pode levar a diagnósticos imprecisos das causas de (in)sucesso dos estudantes, a ideia é adotar uma visão multidimensional, de modo que as informações das escolas sejam correlacionadas com as informações do território em que elas estão inseridas, trazendo à tona informações que ficariam obscuras em uma visão clássica.

A visão multidimensional permite uma leitura integradora do território onde a escola está inserida, de modo a permitir um processo inovador de diagnóstico também baseado na análise de outras diversas variáveis da cidade, tais como as relacionadas a segurança, saúde, empregabilidade dos pais, transporte, etc. Essas variáveis certamente influenciam diretamente na comunidade e, consequentemente, no dia-a-dia dos estudantes, de suas famílias e dos profissionais da educação. Assim, é através desse processo que se consegue chegar ao conceito de território inteligente, que diz respeito à capacidade de analisar, diagnosticar e tomar decisões considerando, de forma integrada, características geográficas, sociais, econômicas e culturais do território [Giovanella 2014].

Com a implementação do conceito de cidade inteligente, tem sido possível observar um uso cada vez mais intensivo de sistemas provendo diferentes dados e serviços com base nos quais é possível tomar decisões estratégicas para o espaço urbano. Contudo, tais cenários são tipicamente caracterizados pela alta heterogeneidade das fontes de dados, $\mathrm{o}$ que acaba dificultando a tarefa de desenvolver de aplicações de valor agregado para os usuários ou produzir novas informações úteis à gestão pública [Naphade et al. 2011]. Para suplantar essas limitações, plataformas computacionais podem ser utilizadas para integrar, de forma transparente às aplicações e/ou usuários, diferentes fontes de informação 
VII Congresso Brasileiro de Informática na Educação (CBIE 2018)

Anais do XXIX Simpósio Brasileiro de Informática na Educação (SBIE 2018)

[Cavalcante et al. 2017]. No caso dos dados educacionais, tais plataformas permitem gerir essas informações provenientes de diversas fontes, além de correlacionar esses dados com fatores não-educacionais, de forma contextualizada.

O objetivo específico deste artigo é apresentar o $S G e o L-E d u c$, uma plataforma que permite integrar dados de diversas fontes educacionais com dados geográficos de uma cidade. A ideia dessa plataforma é, por exemplo, permitir a utilização de informações sobre segurança e saúde para identificar problemas em uma determinada região da cidade e auxiliar na definição de políticas públicas relacionadas a educação por meio da combinação de tais informações com dados educacionais. O SGeoL-Educ disponibiliza ainda ferramentas que podem auxiliar gestores no cruzamento e análise dos dados por ela disponibilizados. Através de visualizações em mapas, é possível ter acesso a informações de diferentes camadas temáticas (educação, saúde, segurança, áreas menos favorecidas economicamente, etc.), fazer consultas cruzando dados de diferentes camadas, criar novas camadas, entre outras funcionalidades.

O restante desse artigo está organizado da seguinte forma. A Seção 2 apresenta o SGeoL-Educ e suas potencialidades que permitem o diagnóstico dos sucessos e insucessos escolares. A Seção 3 ilustra o uso da plataforma proposta neste trabalho no contexto da cidade do Natal, Brasil, mostrando como os dados de sistemas de informação da cidade, da Avaliação Nacional do Rendimento Escolar (ANRESC, conhecida como Prova Brasil), do Sistema de Avaliação da Educação Básica (SAEB) ${ }^{1}$ e de outros sistemas integrados. Os trabalhos relacionados ao SGeoL-Educ são retratados na Seção 4. Por fim, a Seção 5 traz algumas considerações finais.

\section{SGeoL-Educ: Integrando Informações sobre o Território Inteligente}

O SGeoL-Educ é uma plataforma para educação no território inteligente que visa integrar informações geográficas do espaço urbano com dados educacionais providos por diversas fontes, bem como outras informações sobre aspectos importantes da cidade (segurança, saúde, etc.), permitindo pensar a educação em uma perspectiva sistêmica do território inteligente e sustentável. Dessa forma, os territórios passam a ser espaços ativos, isto é, são tidos como o resultado de um campo de forças em que o nível do output depende da capacidade para produzir um misto de coesão, inovação e de comportamentos estratégicos em um contexto sistêmico e evolutivo.

O SGeoL-Educ materializa a ideia da análise integradora e territorializada da educação escolar na qual o território é um fator de regulação entre diferentes membros, grupos e esferas de uma mesma sociedade. Nesse sentido, o precário desempenho dos estudantes na escola pública não se insere e ganha expressão apenas no contorno da sua escola, mas sim vai para muito além desta. Essa leitura integradora e holística da educação situada no território permite identificar fenômenos que tradicionalmente são observados de maneira unidimensional, passando para uma perspectiva multidimensional em que diferentes camadas além da educacional (social, econômica, demográfica, de segurança, cultural, entre outras) conectam-se, interagem e compõem o cenário dos fatores que fragilizam a trajetória escolar de grande parte dos estudantes nas escolas públicas.

A plataforma constitui-se uma ferramenta de suporte para o georreferenciamento de dados de diferentes camadas/fatores inerentes ao território, permitindo uma análise

\footnotetext{
${ }^{1} \mathrm{http}: / /$ portal.inep.gov.br/web/guest/educacao-basica/saeb
} 
VII Congresso Brasileiro de Informática na Educação (CBIE 2018)

Anais do XXIX Simpósio Brasileiro de Informática na Educação (SBIE 2018)

espacial com o manejo de um grupo de variáveis e a interação de dados de diferentes fontes. Além disso, o SGeoL-Educ permite visualizar e especializar os resultados, bem como produzir documentos cartográficos sobre fatores de vulnerabilidades socioeducativas, notadamente aquelas que alcançam maior expressão no contexto escolar situado no território. Essa ferramenta tem ainda o potencial de contribuir para a concretização de mais um desafio, que é fomentar a passagem da escola tradicional para a Educação Escolar do Século XXI, um modelo inovador a ser pautado na necessidade de se criar novos caminhos, com estratégias voltadas para superar o que habitualmente se entende e se faz no campo da educação escolar, inclusive tomando a ciência e a tecnologia como alicerces [Calvo 2016].

O SGeoL-Educ é construído sobre o Smart Geo Layers (SGeoL) [Souza et al. 2017], um middleware que tem a capacidade de integrar os mais diversos tipos de fontes de dados, reunindo uma série de camadas que fornecem um vasto conjunto de informações. O SGeoL utiliza com base a FIWARE ${ }^{2}$, uma plataforma desenvolvida na Comunidade Europeia e utilizada em diversas aplicações para cidades inteligentes nos mais variados cenários. A FIWARE atende a uma ampla gama de requisitos considerados relevantes para cidades inteligentes e provê diversos componentes funcionais genéricos, extensíveis e reutilizáveis que podem facilitar o desenvolvimento de aplicações fazem com que essa plataforma seja uma opção interessante a ser adotada nesse contexto. O SGeoL permite combinar dados que contêm informações geoespaciais como base para integrar dados heterogêneos, provenientes de várias origens, no contexto de uma cidade. Esse middleware organiza e gerencia as informações de uma cidade representando-as na forma de camadas, cada uma delas composta por um conjunto de elementos (entidades) que possuem atributos geoespaciais passíveis de plotagem em um mapa. Por exemplo, uma camada referente às escolas de uma determinada cidade pode ser exibida representando as escolas como pontos marcados no mapa da cidade. Nessa camada, cada entidade (no caso, escola) pode ter atributos como número de professores, número de alunos, índice de evasão, tipo da escola (se de Ensino Fundamental e/ou Ensino Médio, etc.), entre outros.

No SGeoL há dois grupos principais de camadas, as camadas básicas e as camadas processadas. As camadas básicas são responsáveis pelas entidades comumente presentes em modelos de mapeamento urbano, tais como bairros, lotes, quadras, escolas, delegacias, postos de saúde, dados de trânsito, etc. Já as camadas processadas são geradas pela composição de camadas básicas para propósitos diversos. Um exemplo de uma camada processada seria a predição de locais para instalação de novas escolas, resultante da composição das camadas básicas de bairros, escolas e de dados demográficos da cidade, evidenciando bairros com grande densidade demográfica e poucas escolas para atender à demanda.

\section{Aplicação em Natal, Brasil}

O SGeoL-Educ foi instanciado com dados da cidade do Natal provenientes de várias fontes. Para as informações geográficas, a Secretaria Municipal de Meio Ambiente e Urbanismo (SEMURB) da cidade forneceu dados que incluem informações sobre edificações, lotes, favelas, áreas de risco, escolas e creches, áreas verdes, parques, feiras livres, ruas e logradouros, praças, hospitais, zonas administrativas, bairros, conjuntos habitacionais, equipamentos desportivos e orlas. Por sua vez, a Secretaria Estadual da Segurança Pública

\footnotetext{
2 https://www.fiware.org/
} 
VII Congresso Brasileiro de Informática na Educação (CBIE 2018)

Anais do XXIX Simpósio Brasileiro de Informática na Educação (SBIE 2018)

e da Defesa Social (SESED) do Rio Grande do Norte forneceu alguns dados de segurança e índices de ocorrências. Para o levantamento dos dados inerentes a educação na cidade do Natal, foram integrados ao SGeoL-Educ dados provenientes (i) do SIGEduc, sistema da Secretaria Estadual de Educação do Rio Grande do Norte, e (ii) da Anresc/Prova Brasil e do Censo Escolar, estes providos pelo Instituto Nacional de Estudos e Pesquisas Educacionais (INEP).

O INEP, em seu website ${ }^{3}$, disponibiliza para consultas e download informações e estatísticas que ajudam a interpretar a educação em seus variados níveis, como, por exemplo, a Prova Brasil, que tem como objetivo avaliar, a partir de testes padronizados e questionários socioeconômicos, a qualidade do ensino oferecido pelo sistema educacional brasileiro. A integração dos dados da Prova Brasil no SGeoL-Educ permite, assim, usar indicadores que podem prover fatores para produção de diagnósticos. No processo de análise dos dados da Prova Brasil foram verificados os seguintes aspectos:

- A documentação dos dados da Prova Brasil, apesar de disponibilizar um dicionário para cada índice, não apresenta seus respectivos relacionamentos. Por essa razão, foi necessário investigar outros arquivos disponibilizados pelo INEP para que esses critérios fossem encontrados, o que ocorreu nos arquivos do Censo Escolar.

- Os dados da Prova Brasil não contêm a localização espacial das escolas. Para obter esses dados, foi necessário relacionar as escolas através de seus nomes com os dados provenientes do sistema SIGEduc.

Para a identificação dos nomes das escolas e dos municípios a partir dos códigos presentes nos arquivos de escolas da Prova Brasil, foi necessário cruzar dados de outras tabelas de dados do Censo Escolar. De posse dos nomes das escolas e municípios, foram relacionadas as tabelas de dados da Prova Brasil e do SIGEduc, permitindo fazer uso dos dados de ambas e suas localizações geográficas. A Figura 1 ilustra a origem destes dados e a maneira como foram definidas essas associações. Tendo em vista que os dados providos pelo INEP são disponibilizados em arquivos no formato CSV, foram utilizadas facilidades da biblioteca Apache Commons $\mathrm{CSV}^{4}$ para que fosse possível extrair dados a partir de arquivos nesse formato. Os dados do SIGEduc foram obtidos por meio de requisições à interface provida pelo próprio sistema. Em seguida, foi estabelecido um modelo espaço-temporal compatível que, por sua vez, permitiu estabelecer os relacionamentos específicos para as escolas.

Uma vez armazenadas no banco de dados do SGeoL-Educ, é possível utilizar as camadas e suas respectivas propriedades para realizar consultas sensíveis a educação e agregá-las com outras camadas e/ou suas propriedades. As Tabelas 1, 2 e 3 apresentam três diferentes consultas, ressaltando, para cada uma delas, as camadas utilizadas, as propriedades consideradas na consulta e os aspectos que são revelados como resultado da consulta. Por exemplo, uma consulta de análise da distribuição das escolas do Ensino Fundamental no território utiliza três camadas: Escolas, Bairros e Densidade Populacional. As propriedades estabelecem que apenas escolas do Ensino Fundamental devem ser analisadas. O resultado da combinação das três camadas é capaz de revelar os desequilíbrios entre a localização das escolas de Ensino Fundamental e a concentração demográfica, como mostra o mapa da Figura 2.

\footnotetext{
${ }^{3}$ http://portal.inep.gov.br

${ }^{4} \mathrm{https}$ ///commons.apache.org/proper/commons-csv/
} 
VII Congresso Brasileiro de Informática na Educação (CBIE 2018)

Anais do XXIX Simpósio Brasileiro de Informática na Educação (SBIE 2018)

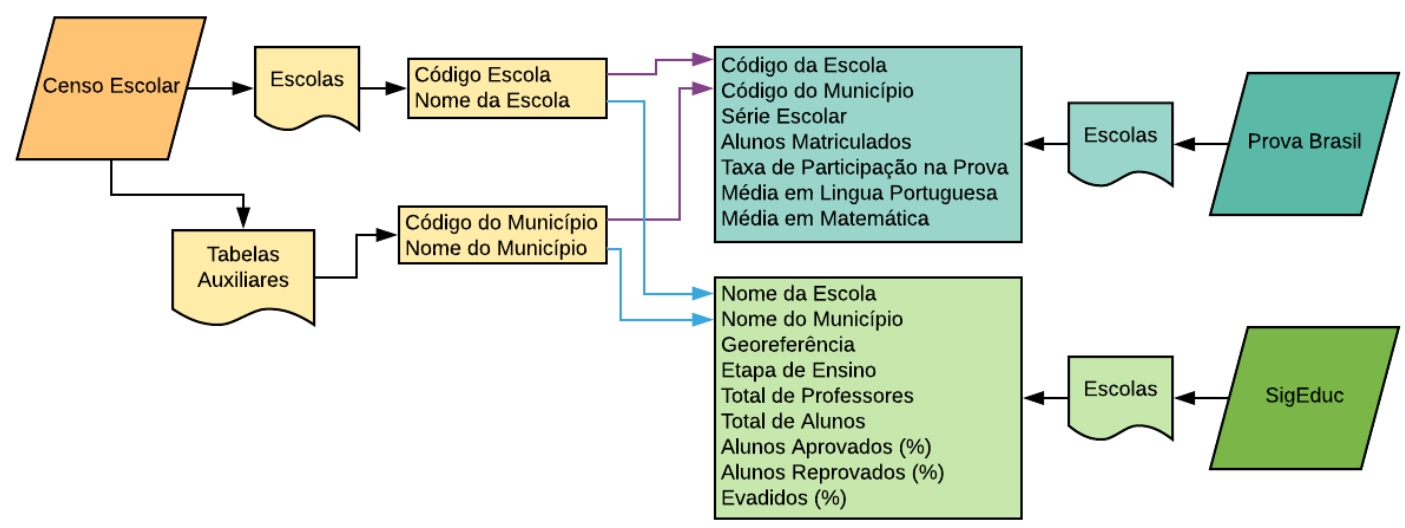

Figura 1. Associação dos dados do INEP (Censo Escolar e Prova Brasil) e do SIGEduc.

Tabela 1. Consulta no SGeoL-Educ para analisar a distribuição de escolas de Ensino Fundamental no território considerando a densidade demográfica.

\begin{tabular}{|l|l|l|l|}
\hline Consulta & $\begin{array}{l}\text { Camadas } \\
\text { utilizadas }\end{array}$ & $\begin{array}{l}\text { Propriedades } \\
\text { utilizadas }\end{array}$ & Resultado \\
\hline $\begin{array}{l}\text { Análise da distribuição das } \\
\text { escolas de Ensino Fundamen- } \\
\text { tal no território }\end{array}$ & Escolas & $\begin{array}{l}\text { Ensino } \\
\text { Fundamental }\end{array}$ & $\begin{array}{l}\text { Desequilíbrios entre a localização das } \\
\text { escolas de Ensino Fundamental e a lo- } \\
\text { calização de suas demandas (concen- } \\
\text { tração demográfica) }\end{array}$ \\
\cline { 2 - 3 } & Bairros & - & \\
\cline { 2 - 4 } & $\begin{array}{l}\text { Densidade } \\
\text { Populacional }\end{array}$ & - & \\
\hline
\end{tabular}

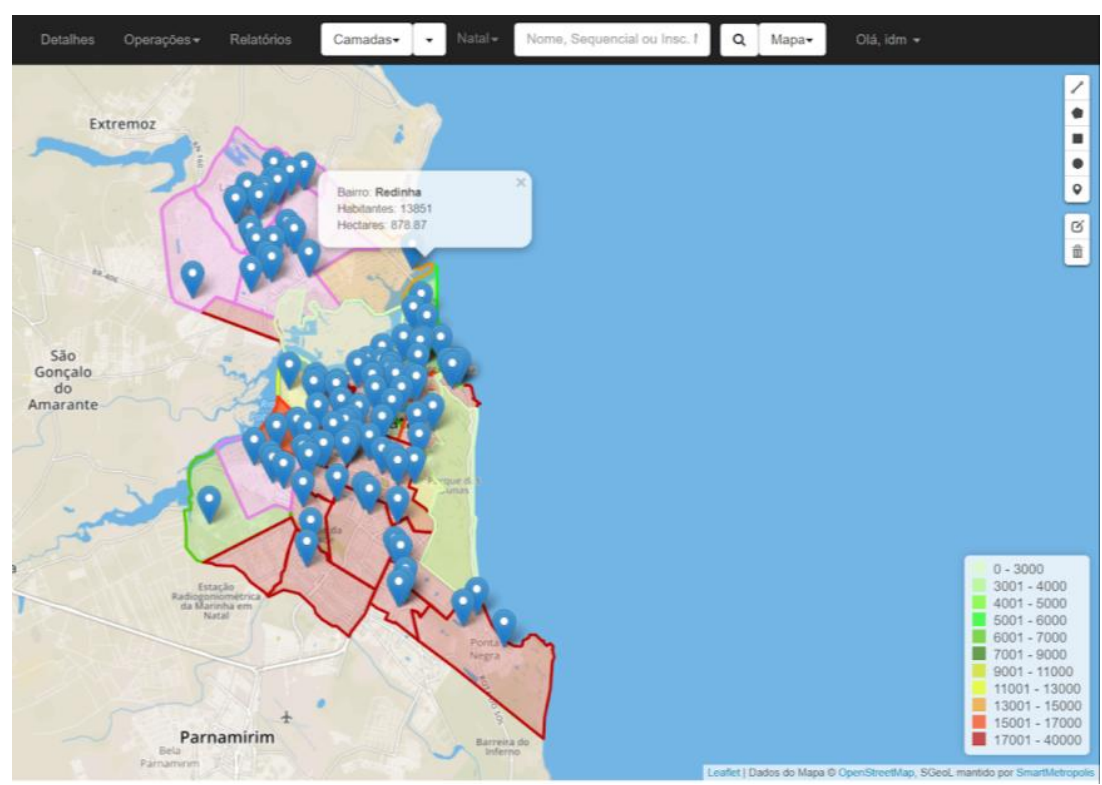

Figura 2. Interface do SGeoL-Educ mostrando no mapa desequilíbrios entre a localização das escolas de Ensino Fundamental e a localização de suas demandas (concentração demográfica). Os pinos indicam a localização das escolas e os polígonos indicam os bairros da cidade de acordo com uma escala populacional. 
VII Congresso Brasileiro de Informática na Educação (CBIE 2018)

Anais do XXIX Simpósio Brasileiro de Informática na Educação (SBIE 2018)

Um segundo exemplo de consulta tem a finalidade de identificar escolas de Ensino Fundamental que têm baixa proficiência em Matemática (considerando os índices estabelecidos no SAEB) no $9^{\circ}$ Ano do Ensino Fundamental. Para isso é possível usar as camadas Escolas e Zonas. As propriedades da consulta especificam que somente escolas localizadas Zona Norte da cidade do Natal com proficiência em Matemática abaixo do Nível 3 nos níveis finais do Ensino Fundamental devem ser consideradas. Como resultado, o mapa como o mostrado na Figura 3 apresentaria a localização das escolas da Zona Norte da cidade cujos estudantes têm dificuldades na aprendizagem de Matemática.

Tabela 2. Consulta no SGeoL-Educ para identificar escolas de Ensino Fundamental com baixa proficiência em Matemática no 9ํ Ano.

\begin{tabular}{|c|c|c|c|}
\hline Consulta & $\begin{array}{l}\text { Camadas } \\
\text { utilizadas }\end{array}$ & $\begin{array}{l}\text { Propriedades } \\
\text { utilizadas }\end{array}$ & Resultado \\
\hline \multirow[t]{2}{*}{$\begin{array}{l}\text { Identificação de escolas com } \\
\text { baixa proficiência em Mate- } \\
\text { mática no } 9^{\circ} \text { Ano do Ensino } \\
\text { Fundamental }\end{array}$} & Escolas & $\begin{array}{l}\text { Proficiência em Matemá- } \\
\text { tica menor que o Nível } \\
3(<200) \\
9^{\circ} \text { Ano }\end{array}$ & \multirow{2}{*}{$\begin{array}{l}\text { Visualização da localização } \\
\text { das escolas da Zona Norte da } \\
\text { cidade do Natal com baixa } \\
\text { proficiência em Matemática } \\
\text { no ano final do Ensino Fun- } \\
\text { damental }\end{array}$} \\
\hline & Zonas & Zona Norte & \\
\hline
\end{tabular}

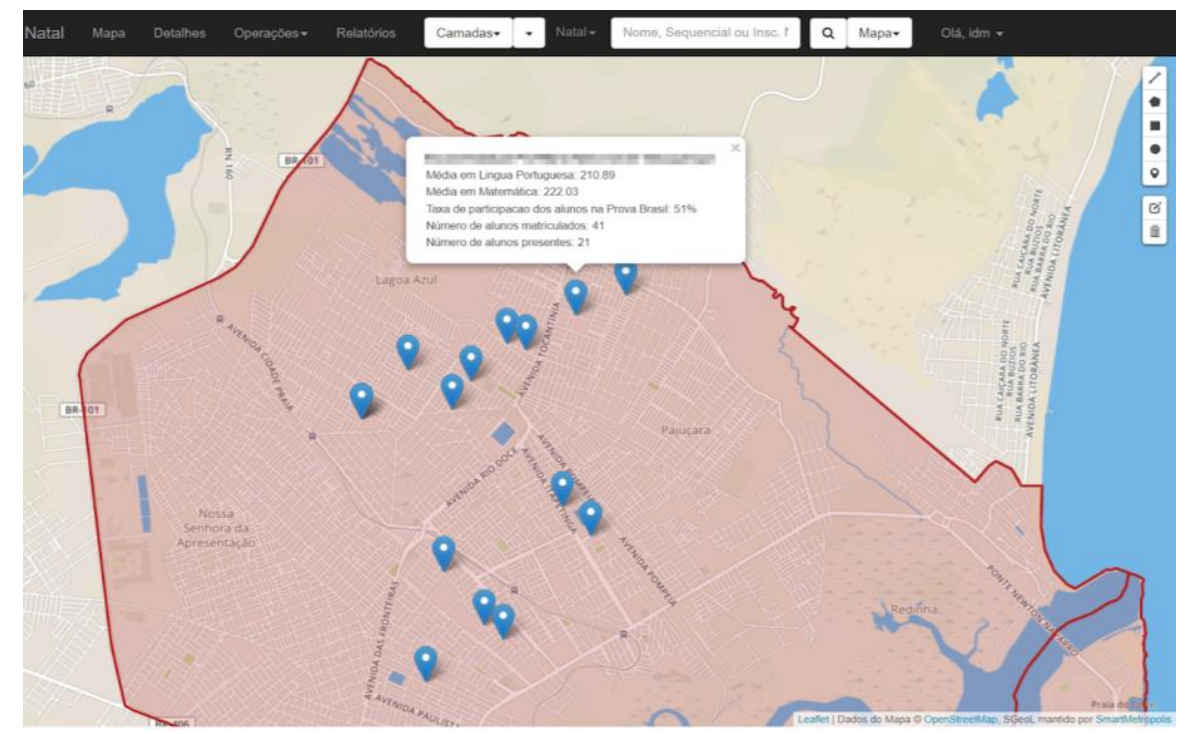

Figura 3. Interface gráfica com a localização das escolas (pinos) na Zona Norte de Natal (área demarcada) cujos estudantes do 9 Ano do Ensino Fundamental apresentam baixa proficiência em Matemática.

Por fim, uma terceira consulta poderia identificar escolas próximas a zonas de violência, deslizamentos, favelas, praças, áreas com equipamentos desportivos e áreas verdes. Como mostra a Tabela 3, sete camadas diferentes são usadas nessa consulta. $\mathrm{O}$ resultado mostra a localização das escolas confrontada com áreas que têm relação direta com a qualidade de vida da população, ocupação do solo, infraestrutura e segurança pública. De maneira similar, outras consultas (inclusive mais elaboradas) poderiam revelar outras realidades do contexto educacional no território. 
VII Congresso Brasileiro de Informática na Educação (CBIE 2018)

Anais do XXIX Simpósio Brasileiro de Informática na Educação (SBIE 2018)

Tabela 3. Consulta no SGeoL-Educ para identificar escolas próximas a áreas de violência, de risco e de lazer.

\begin{tabular}{|l|l|l|l|}
\hline Consulta & Camadas & Propriedades & Resultado \\
\hline $\begin{array}{l}\text { Identificação de esco- } \\
\text { las próximas a áreas de } \\
\text { violência, de risco e de } \\
\text { lazer }\end{array}$ & Escolas & Pública & $\begin{array}{l}\text { Visualização da localização das } \\
\text { escolas próximas a áreas com } \\
\text { ocorrências de segurança, áreas de } \\
\text { risco de deslizamento, e áreas com } \\
\text { praças, equipamentos desportivos } \\
\text { e verdes }\end{array}$ \\
\cline { 2 - 3 } & Segurança & $\begin{array}{l}\text { Tráfico de drogas } \\
\text { Assaltos } \\
\text { Agressões } \\
\text { Postos policiais }\end{array}$ & \\
\cline { 2 - 3 } & Áreas de Risco & Deslizamentos & \\
\cline { 2 - 3 } & Favelas & - & \\
\cline { 2 - 3 } & Praças & - & \\
\cline { 2 - 3 } & $\begin{array}{l}\text { Equipamentos } \\
\text { Desportivos }\end{array}$ & - & \\
\cline { 2 - 3 } & Áreas Verdes & - & \\
& & & \\
\cline { 2 - 3 } & & & \\
\hline
\end{tabular}

\section{Trabalhos Relacionados}

A literatura reporta diversos trabalhos relacionados ao desenvolvimento de ferramentas de apoio à tomada de decisão com base na integração e análise de dados educacionais. Alvares et al. (2015) desenvolveram uma aplicação de descoberta de dados que permite que o usuário tenha acesso aos dados dos cursos participantes do Exame Nacional de Desempenho de Estudantes (Enade) dos cursos de Ensino Superior no Brasil. A metodologia utilizada para o desenvolvimento da aplicação foi a de extração, transformação e carregamento de dados (ETL - Extract-Transform-Load) do Enade, disponibilizados pelo INEP, e de estados e regiões do Brasil, providos pelo Instituto Brasileiro de Geografia e Estatística (IBGE). Em seguida, foi realizada a integração desses dados e desenvolvida uma interface para apresentação dos dados para o usuário. A integração de dados do Enade e do IBGE permite avaliar os resultados do exame desde um nível mais amplo até a realização de consultas mais detalhadas como o desempenho de um curso em particular. Contudo, abordagens ETL possuem desvantagens tais como a necessidade frequente da intervenção humana principalmente na etapa de transformação dos dados. De forma similar, Pinheiro et al. (2013) desenvolveram uma aplicação Web para análise do resultado do desempenho escolar dos alunos com base nos resultados da Prova Brasil dos anos de 2007, 2009 e 2011, disponibilizados pelo INEP. A aplicação permite a realização de consultas de informações dos estudantes em relação ao desempenho escolar, habilidades adquiridas, relação dos dados socioeconômicos com os resultados alcançados na escola, etc.

Sousa et al. (2015) desenvolveram um sistema Web de apoio a pesquisa em educação utilizando dados do INEP para a realização de consultas que permitem, por exemplo, aplicar políticas específicas para alguma área com alto índice de evasão escolar, prever a possível falta de professores, monitorar o progresso dos estudantes, entre outras possibilidades. Já Santos et al. (2017) usam técnicas de mineração de dados para análise 
VII Congresso Brasileiro de Informática na Educação (CBIE 2018)

Anais do XXIX Simpósio Brasileiro de Informática na Educação (SBIE 2018)

das declarações dos estudantes em avaliações institucionais docentes a fim de verificar a relação entre as abordagens pedagógicas utilizadas pelos professores e o nível de aprendizado do estudante. Outra ferramenta para análise de dados educacionais é o T-Partner, um software que faz uso de Inteligência Computacional para auxiliar o instrutor na tomada de decisões pedagógicas em ambientes de aprendizagem on-line [Paiva et al. 2017]. Os estudantes interagem com um ambiente de aprendizado e o T-Partner realiza a coleta dos dados gerados a partir dessa interação, processando tais dados e informando os instrutores sobre situações de interesse educacional. No entanto, uma limitação identificada é o fato de o sistema utilizar apenas os dados gerados pela interação dos estudantes no ambiente de aprendizagem, não permitindo a importação de novas fontes de dados para realizar possíveis integrações de dados.

Apesar de os trabalhos mencionados facilitarem, de certa forma, a compreensão de determinados aspectos da educação, as consultas disponibilizadas por essas ferramentas são limitadas. Um grande diferencial do SGeoL-Educ em relação a essas propostas existentes diz respeito justamente às diversas possibilidades de relacionamento/cruzamento de dados, tendo em vista que a ferramenta integra não apenas dados educacionais, mas também dados importantes de diversos domínios, como saúde, segurança, transporte, infraestrutura etc., o que proporciona uma visão mais abrangente dos aspectos que influenciam no aprendizado dos estudantes e na educação como um todo. Outra vantagem da plataforma é a disponibilização de uma interface para importação dos dados, o que facilita o processo de extração dos dados das diversas fontes de dados existentes.

\section{Considerações Finais}

A plataforma SGeoL-Educ integra dados georreferenciados e informações educacionais permitindo uma análise integrada, multidimensional e territorializada da educação escolar. A plataforma integra dados heterogêneos, disponibiliza visualizações de informações em mapas e permite fazer consultas cruzando dados de várias fontes, espacializando os resultados das consultas, composições e produção de informações acerca de fatores de vulnerabilidades socioeducativas. A instanciação do SGeoL-Educ para a cidade do Natal integrou dados geográficos, dados de segurança e informações de desempenho escolar, todos provenientes de fontes distintas. Tal instanciação comprovou o potencial de integração e a expressividade da ferramenta, materializando assim a possibilidade de se realizar uma leitura integradora e holística da educação situada no território. Com isso, é possível identificar fenômenos em uma perspectiva multidimensional em que diferentes camadas de informação (incluindo educacionais), conectam-se, interagem e compõem o cenário dos fatores que fragilizam a trajetória escolar de grande parte dos estudantes das escolas públicas no Brasil.

\section{Referências}

Alvares, R. V., Campos, N. S., Gomes, V. B. (2015) “Adoção de Data Discovery para apoio ao processo de análise de dados do Enade”. In: Sánchez, J. (org.) Memorias del XX Congreso Internacional de Informática Educativa. Nuevas Ideas en Informática Educativa 11, pp. 480-485.

Calvo, A. H. (2016) Viagem à escola do Século XXI: Assim trabalham os colégios mais inovadores do mundo. São Paulo, SP, Brasil: Fundação Telefônica Vivo. 
VII Congresso Brasileiro de Informática na Educação (CBIE 2018)

Anais do XXIX Simpósio Brasileiro de Informática na Educação (SBIE 2018)

Cavalcante, E., Cacho, N., Lopes, F., Batista, T. (2017) "Challenges to the development of smart city systems: A system-of-systems view", Proceedings of the 31st Brazilian Symposium on Software Engineering. New York, NY, USA: ACM, pp. 244-249.

Giovanella, C. (2014) "Smart territory analytics: Toward a shared vision", Proc. of the 47th Scientific Meeting of the Italian Statistical Society. Cagliari, Italy: CUEC.

Naphade, M., Banavar, G., Harrison, C., Paraszczak, J., Morris, R. (2011) "Smarter cities and their innovation challenges", Computer 44(6), pp. 32-39.

Paiva, R., Bittencourt, I. I., Lima, A., Amorim, S., Lemos, W., Dermeval, D., Isotani, S. (2017) "Usability perspective of an authoring solution to assist pedagogical decisionmaking”, Anais do XXVIII Simpósio Brasileiro de Informática na Educação. Porto Alegre, RS, Brasil: SBC, pp. 1587-1596.

Pinheiro, R. G. P., Elia, M., Sampaio, F. F. (2013) "Avaliando as competências escolares através da Prova Brasil usando ferramenta web", Anais do $19^{\circ}$ Workshop de Informática na Escola. Porto Alegre, RS, Brasil: SBC, pp. 60-69.

Santos, F. P., Silveira, I. F., Lechugo, C. P. (2017) “Análise da percepção dos alunos sobre as práticas docentes por meio da mineração de dados educacionais", Revista Espacios 38(5).

Sousa, R., Silva, L. E. P. (2015) "BRAVO Sistema Web de apoio à pesquisa em Educação", Anais dos Workshops do IV Congresso Brasileiro de Informática na Educação. Porto Alegre, RS, Brasil: SBC, pp. 105-114.

Souza, A. et al. (2017) "A data integration approach for smart cities: The case of Natal", Proceedings of the 2017 International Smart Cities Conference. USA: IEEE. 